\title{
Emigración y cambio de actividad en el Pirineo
}

\author{
M. ${ }^{2}$ Teresa Rubio Benito
}

\section{INTRODUCCIÓN}

La montaña tiene hoy un gran protagonismo, derivado de su situación crítica, en virtud de la decadencia de sus actividades tradicionales, que ha determinado un estancamiento, cuando no, un retroceso de su economia.

En líneas generales la política de montaña de la CEE como las específicas de los Estados de Europa del W., van encaminadas a asegurar la continuidad de la actividad agraria en estas zonas, para mantener unos mínimos poblacionales, salvaguardar el medio natural (protección contra la erosión principalmente) y responder a las necesidades de ocio que de ellas se demanda.

En los últimos 40 años, las montañas pirenáicas y centroeuropeas han sufrido un fuerte proceso de transformación funcional; en este cambio, la agricultura ha sido a veces desplazada de su lugar como actividad principal y frecuentemente única en la montaña, a un lugar secundario, a veces marginal. (Majoral 1986).

Actualmente aunque su importancia haya decaído, no puede hablarse de las dificultades de los espacios de montaña desde un punto de vista únicamente agrícola, sino de espacios multifuncionales en los que la agricultura se desarrolla en relación con otras actividades, paralela o

\footnotetext{
* Departamento de Geogratía. Universidad Nacional de Educación a Distancia.
} 
integradamente. Problemas de los Pirineos, tales como la emigración de la población y su concentración en los valles, desaparición de situaciones de subsistencia y regresión de los movimientos trashumantes, son comunes en la mayor parte de la montaña centroeuropea Alpes, Cárpatos, Jura o Macizo Central francés.

Esta situación fue precedida en una parte del espacio europeo por una fase de sobreexplotación de la montaña que se tradujo primordialmente, tanto en una extensión del área cultivada y en el aumento de carga ganadera (con el consiguiente agotamiento de los pastos), como en la presión del hombre sobre los recursos forestales que retrocedieron notablemente en función de sus necesidades energéticas y de vivienda.

Hasta los años 50, puede decirse que la montaña templada funcionaba como un sistema relativamente en equilibrio, basado en una economía de subsistencia dentro de un sistema de relaciones cerrado. La autonomía relativa de los valles, reposaba sobre una trilogía: la explotación del bosque y de las minas, el ganado y las industrias complementarias de transformación. (Dézert 1975).

A partir de esa década, el desarrollo industrial generalizado, supuso la maquinización y la sustitución de los medios de tracción animal por tractores, lo que significó la liquidación definitiva de la cabaña equina y también, un fuerte éxodo rural favorecido no sólo por la demanda de mano de obra industrial, (Noin 1983), sino también por la transformación en regadio de grandes zonas del llano. La desaparición de montes y pastizales al roturar nuevas tierras e introducir nuevos cultivos, acentuó la crisis del sistema pastoril en régimen de trashumancia. (Boserup 1984).

En realidad, el proceso tiene sus raíces en el cambio económico operado en el siglo XIX, en el que se produjo un proceso de disolución de las antiguas formas de explotación feudal a favor de los objetivos de una sociedad industrial.

\section{INDUSTRIA Y EMIGRACIÓN}

Desde finales del siglo pasado hasta la segunda guerra mundial, la hidroelectricidad permitió a la gran industria moderna asentarse al pie de los grandes valles pirenáicos, gracias a la iniciativa empresarial lanzada 
desde los centros urbanos regionales. Es lo que Dézert (1975) considera la tercera fase económica en la evolución de la vida industrial en la montaña.

La clave del éxito radicaba en buena medida, en la obtención de una energía barata, aprovechando los caudales fluviales de los vasos naturales y el desnivel topográfico existente en el salto de agua. La mayoría de los embalses se construyeron bajo una óptica de grandes obras, vinculadas a economías de escala, pero no se contemplaron las pequeñas presas de regulación de cabecera; de esta forma, se perdió una riqueza susceptible de ser empleada en los altos valles intramontañosos, para regar los pastos de verano. (Molina 1986).

En la montaña el agua no es sólo un recurso biológico, sino también un recurso económico y tecnológico de gran trascendencia; sin embargo, no ha sido motor de desarrollo en estos espacios, quizá porque tampoco se ha realizado una auténtica planificación en relación con sus usos.

Su escasa incidencia en el despegue económico del Pirineo, obedece, posiblemente a tres hechos: baja utilización de su potencial de desarrollo, subutilización de recursos, o uso de los mismos con vistas al impulso de otros espacios económicos ajenos a esa zona, sin que haya revertido positivamente en la economía montañesa.

La ubicación de los embalses y su tamaño, produjo un gran impacto en el medio rural, ya que se anegaron los fondos de los valles donde se localiza prácticamente el total de la superficie labrada y una parte importante de la agraria útil. (Monserrat y Fillat 1979). Este hecho, repercutió directamente en la disminución de la producción de forrajes e indirectamente en la ganadería.

Al anegar los prados y tierras de labor del valle, arruinaron la propiedad ya que los prados situados en la montaña, con dificultades de acceso debido a la ruptura de comunicaciones entre las dos orillas, no cubrian las necesidades mínimas para mantener el ganado vacuno y asegurar su estabulación en invierno; de esta forma, los propietarios se vieron forzados indirectamente a emigrar (Daumas 1976).

Si el fenómeno de la emigración no era nuevo en el Pirineo, ya que se venía arrastrando desde el siglo XIX ante la falta de oficios supletorios, sí fue nueva la forma masiva en que se produjo a partir de los años 60 $y$ las consecuencias de despoblamiento y de envejecimiento irreversible a las que llegó la población, de tal forma, que la descapitalización humana, fue todavía más dura que la económica, si cabe, aunque ambas en conjunto, llevaron a un cambio de la población activa. (Puyol 1979). 
La fuga de la población protagonizada en principio por las mujeres y generalizada posteriormente al resto de la población, indiscriminadamente, elevó el contingente de población vieja de manera espectacular porque los jóvenes, de forma selectiva, emigraron. (Rodríguez 1985).

En efecto, simultáneamente a la construcción de las obras hidráulicas comentadas, se inició la transformación del suelo agricola en industrial y por tanto, el cambio de actividad económica para muchos habitantes del Pirineo. Según Gabert y Guichonnet (1965) la fuerte densidad de población, fenómeno general en las montañas europeas y asiáticas, proporcionó mano de obra barata, hecho que, unido a la abundancia de agua, aseguró un desarrollo apoyado en las vías naturales de comunicación que indujo a reservar para los valles, las industrias del aluminio. Instalaciones de este tipo se ubicaron en ambas vertientes pirenáicas, proporcionando en pocos años, los trasvases humanos más espectaculares campo-ciudad.

El crecimiento acelerado de Sabiñánigo, lugar con 280 habitantes en el alto valle del río Gállego, convertido hoy en ciudad industrial de 12.000 habitantes, tiene esta explicación y nos sirve de ejemplo aquí. La construcción de una estación de ferrocarril a principios de siglo y la instalación de pequeños centros comerciales y de servicio en torno a la misma, dieron lugar a la creación de un núcleo compacto de población, situado a $1 \mathrm{~km}$ del núcleo primitivo, constituyendo un barrio con entidad propia que pronto multiplicó sus efectivos poblacionales, con la instalación de empresas industriales de capital extranjero, atraído por la facilidad y bajo coste de la energía y por la accesibilidad al ferrocarril. (Rubio y Castelló 1986).

La primera empresa instalada en Sabiñánigo fue Energía e Industrias Aragonesas, sociedad constituída en abril de 1918 con un capital de tres millones de pesetas, aportado mayoritariamente por un grupo francés relacionado con empresas de electroquímica, aluminio e hidroelectricidad, que puso en funcionamiento, en 1923, la primera fábrica de obtención de amoníaco a partir del hidrógeno electrolítico complementando, dos años más tarde estas instalaciones, con industrias de clorato, ferromanganeso, explosivos y carburos.

Además, para intensificar sus fabricaciones clásicas de productos electroquímicos, proporcionó la base para que se establecieran como filiales, una serie de empresas como: Desarrollo Químico Industrial, S. A. (DEQUISA), Fósforos del Pirineo, S. A. y Aragonesas Promoción de Obras y Construcciones. A partir de los años sesenta, en colaboración con la Junta de Energía Nuclear, se montaron las instalaciones necesarias para la recuperación del agua pesada, procedente de las baterías de 


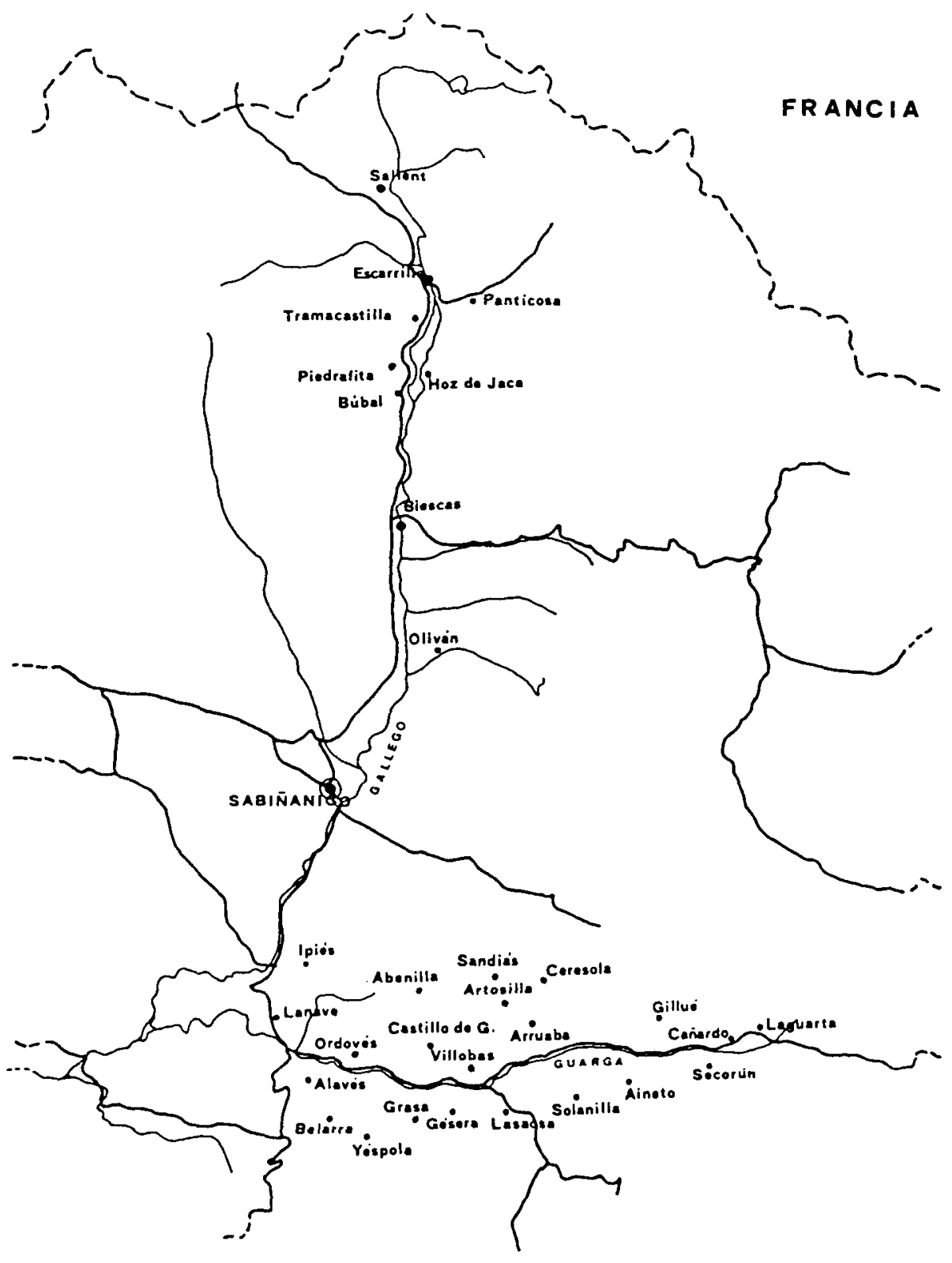

VALLE DE GUARGA 
electrolisis convirtiéndose de esta forma en empresa avanzada en el campo de la energía nuclear.

La Sociedad de Aluminio Español que comienza a funcionar en 1925, también con capital francés, transformando la bauxita del país vecino, representa en la historia de la industrialización de Sabiñánigo un jalón importante en la transformación de la actividad económica de la zona circundante y en definitiva, del paisaje. Al inicio de los años treinta, se había producido ya una ampliación de la producción y del capital, controlado para entonces por el Banco Urquijo al igual que la empresa Energía e Industrias Aragonesas. Aluminio Española incrementó su producción y estableció la gran planta manufacturera de Aluminio Transformación, S. A. y de Earle Aluminio Español, S. A., con fuertes inversiones que más tarde pasaron a denominarse Aluminio de Galicia, sociedad en la que sigue siendo mayoritario el capital francés, ahora en manos de la multinacional Pechiney.

Entre los años cincuenta a setenta, Sabiñánigo vivió un progresivo desarrollo industrial, creándose más de 1.700 puestos de trabajo, ocupados fundamentalmente por vecinos del entorno próximo, bien de los municipios ubicados en el eje del Gállego, bien en el de sus afluentes, como el río Basa y el río Guarga, manteniendo a veces en los mismos una agricultura a tiempo parcial, actividad que fue definitivamente abandonada años más tarde, de tal forma que bastantes municipios dentro del área de influencia de Sabiñánigo, han quedado totalmente despoblados o con una población a punto de extinguir.

El valle de Guarga, situado sobre el río del mismo nombre, afluente del Gállego por su margen izquierdo, mantenía prácticamente una economía de autoabastecimiento con aprovechamiento agropastoril. Consultados los Censos de población desde 1900 hasta 1986, comprobamos que el número de habitantes siempre fue muy bajo, y que no se siguió el mismo criterio para su recuento, diferenciándose entre población concentrada y hábitat disperso, en edificaciones diseminadas.

De los 1.339 habitantes de principios de siglo, 856 estaban clasificados en el primer supuesto y 483 , en el segundo. Secorún, Aineto y Gésera en la margen izquierda y Laguarta en la derecha, eran los núcleos de mayor vitalidad relativa al conjunto, alcanzando entre 100 y 110 habitantes. Entre 1900 y 1950, la zona perdió el $50 \%$ de la población, que emigró a Sabiñánigo atraída por las nuevas instalaciones industriales y los servicios que el apogeo de la ciudad, generaba. Con la primera emigración, se rompió el círculo cerrado de relación que mantenía el $99 \%$ de los habitantes, como reflejan el inmovilismo de los apellidos 
EVOLUCIÓN DE LA POBLACIÓN EN LA GUARGUERA

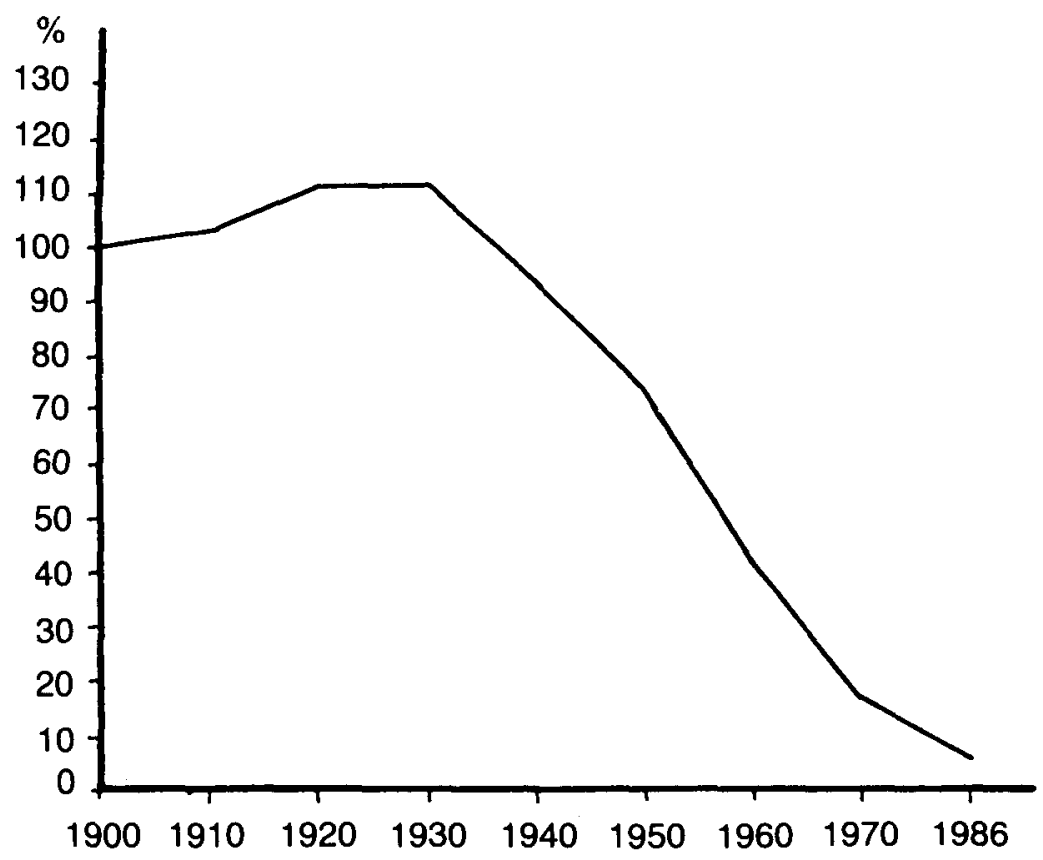

repetidos muchas veces, desapareciendo a la vez oficios típicos como cuchareros, vigueros, sogueros, herreros o guarnicioneros, tan necesarios para la comunidad. Una vez roto este equilibrio de relaciones, la sociedad según Gaviria (1976) se deshace.

A partir de la década de los cincuenta, ya no existe la dualidad entre hábitat concentrado y disperso porque todo el valle se agrupa territorialmente en dos términos: Laguarta y Gésere. El primero se mantiene con 112 habitantes, pero el segundo sólo alcanza 42; para entonces, ya han desaparecido Sandiás en la margen derecha y Alavés en la izquierda, bajando el conjunto poblacional a 670 habitantes, en continuo descenso en años siguientes, hasta el final definitivo observado personalmente en 1986. (Rubio y Castelló 1986).

Puede que en términos absolutos, la desaparición total de estos pequeños núcleos de población o la baja densidad que se mantiene en muchas zonas deprimidas del Pirinero, sea la óptima y que el proceso migratorio iniciado a principios de siglo y precipitado a partir de los años 1955-1965, haya servido de regulador demográfico, expulsando los ele- 
FREC. ABS.

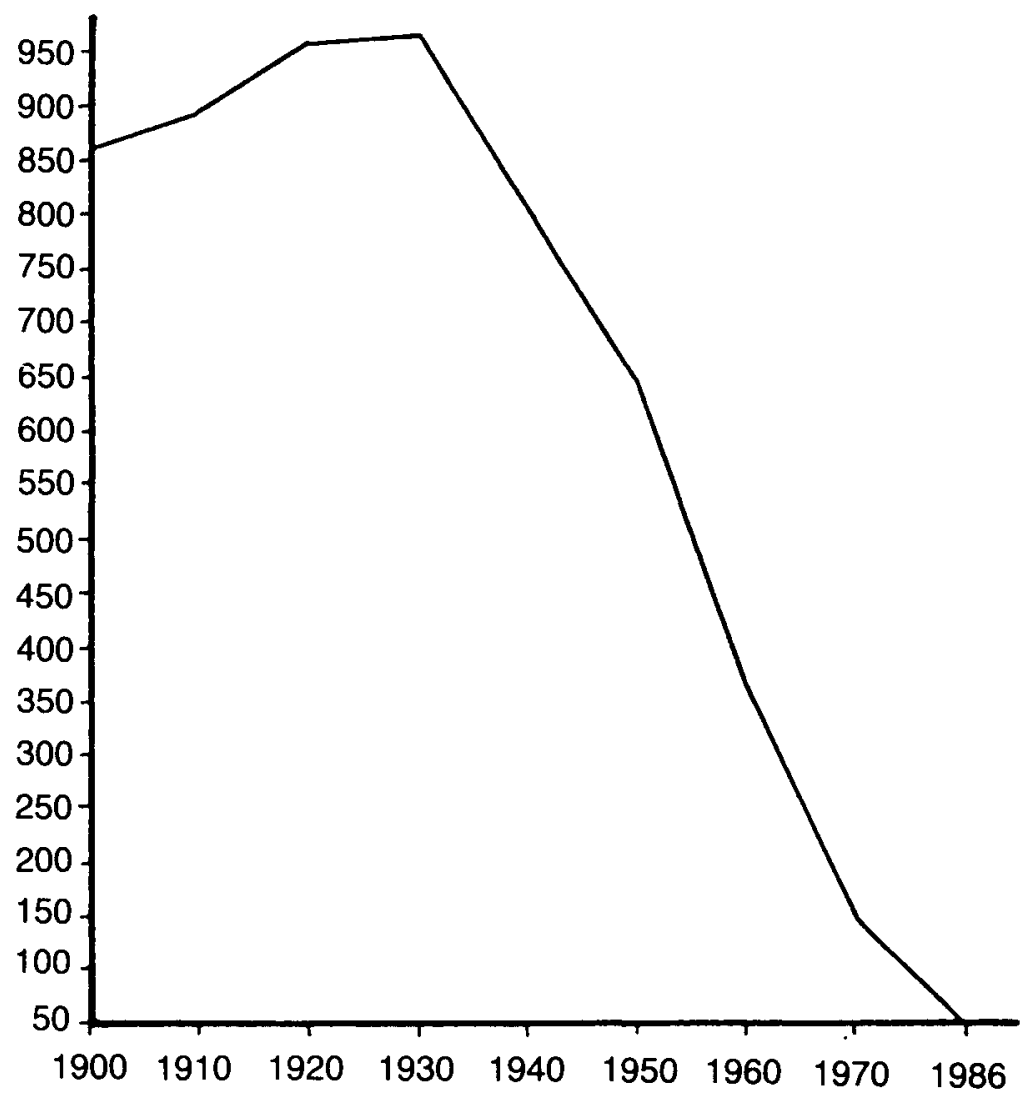

mentos sobrantes de una sociedad tradicional, en retroceso ante el choque brusco con una sociedad industrial. En cualquier caso, la emigración ha supuesto un cambio de actividad económica, casi revolucionario para la montaña y el proceso de readaptación, que se da con distinta cronología, según zonas, todavía presenta contradicciones importantes. (Chadefaud y Dalla Rosa 1978).

Con la pérdida de población joven, la agricultura del Pirinero se adaptó a las necesidades de la ganadería, sustituyendo los policultivos tradicionales de base cerealista, por la producción de forrajes para el ganado (Dalla Rosa 1980); mientras, la desaparición o disminución de la cabaña ganadera, incidió también en los aprovechamientos agrícolas y en la organización de las explotaciones, de forma que, sin capitalizar las 
instalaciones, los pueblos más vitales, mejor comunicados y con mayores perspectivas turísticas, adaptaron el tamaño de las explotaciones a la medida de sus posibilidades, manteniéndolas a tiempo parcial y enlazando directamente con el sector de servicios impulsado poderosamente por el turismo, cada vez más numeroso. (Rubio y Castelló 1987).

La agricultura a tiempo parcial es un nuevo elemento diferenciador del espacio agrícola, no sólo por el cambio que supone en las características de la población que trabaja en la agricultura, sino también porque ayuda a su capitalización y continuación. La regresión del número de explotaciones, es inferior en las áreas donde hay una doble actividad que complemente los ingresos de unas explotaciones, claramente insuficientes en una alta proporción.

Generalmente, son actividades relacionadas con el sector terciario, las que sirven de apoyo económico en la breve estación estival y a veces, también en invierno, en aquellos núcleos privilegiados que cuentan con estaciones de Ski. (Pisano 1986). Efectivamente, ni el paisaje, ni la caza, ni la pesca, pueden compararse en importancia económica a la generada por la nieve, que es el sujeto físico de usos recreativo-deportivos, además de reserva de agua para aprovechamientos ulteriores, en los sectores de producción primario y secundario. Un rasgo fundamental, polémico para algunos autores, es el de su limitada localización como estación de nieve para usos recreativos, que conlleva un alto grado de concentración, no sólo en cuanto a instalaciones se refiere, sino sobre todo, en los efectos que produce sobre el entorno. (Puigdefábregas 1980).

Para los ecologistas a ultranza, la nieve provoca una de las mayores presiones sobre la montaña, con efectos más irreversibles y profundos, por la naturaleza y amplitud de sus impactos, sobre el "paisaje agrario" ya que las estaciones de invierno se localizan en el dominio de los pastos alpinos y generan en torno a ellas la venta de parcelas para la construcción de chalets, sobre la "población", que aumenta en los núcleos más afectados por la estación y se adapta a la nueva situación, según la composición de la familia, que acopla el tamaño de las explotaciones a sus posibilidades, variando también su orientación, ya que se suprime el ganado de ordeño más complicado, reconvirtiendo la explotación en ganado de carne. (López de Sebastián 1981).

Posturas moderadas, entre la que nos encontramos, defienden que el turismo de invierno y la actividad ganadera que desarrollan los municipios afectados por estaciones de nieve, son complementarios sensu estricto, ya que ocupan el mismo espacio físico, en estación climática 
diferente, sin entrar en colisión sus intereses, aunque efectivamente, las instalaciones hoteleras y urbanizaciones residenciales, ocupen espacios dedicados antes a pastos, a veces abandonados a partir de la crisis del campo de 1950. (Rubio y Castelló 1987).

\section{¿TURISMO COMO SOLUCIÓN?}

Los servicios turísticos de la época invernal, los cubren estaciones de nieve, ubicadas a lo largo del Pirineo, en ambas vertientes, aunque existe un enorme contraste entre la proliferación de estos espacios de ocio en Francia y la desigual y tímida iniciativa española. Así, Les Pyrénées-Atlántiques, Hautes Pyrénées, Haute-Garonne, Ariège y les Pyrénées-Orientales, agrupan a 16 estaciones de Ski mientras en España, sólo dos Comunidades Autónomas (Aragón y Cataluña) cuentan con diez instalaciones de este tipo.

Super-Barèges, La Mongie, Saint-Lary, o Gourette y Aubisque, son algunas de las más afamadas estaciones francesas, situadas por encima de los $2.000 \mathrm{~m}$ de altura, con un poder de atracción de cientos de miles de clientes en cada jornada esquiable, según Chadefaud y Dalla Rosa (1978).

Astún, Formigal, Cerler, La Molina y Baqueira-Beret, destacan en el Pirineo español, aunándose verdaderos complejos turísticos, equipados con modernos establecimientos industriales, con otros de carácter familiar, buscando una complementariedad entre invierno y verano en pro de conseguir un mejor aprovechamiento de la infraestructura y servicios generales, cuya génesis y posterior desarrollo, han conseguido variar la estructura ocupacional de la población, provocando un fuerte proceso de aculturación y cambios considerables en el modo de vida de los habitantes de los altos valles pirenáicos.

No obstante, los logros económicos conseguidos en los municipios del Pirineo son parciales, dado el bajo número de estaciones de nieve en funcionamiento y su escasa repercusión en el poder adquisitivo de sus habitantes. La mayor parte de las instalaciones deportivas dependen de sociedades, capital e intereses ajenos a la montaña; este parcial crecimiento inducido debería de sustituirse por un desarrollo endógeno que asegurase la revalorización del espacio rural en el Pirineo. 
No basta con ayudas y subvenciones parciales únicamente en un sector productivo, ya que eso supone dilapidar la inversión; es preciso invertir en infraestructura y servicios sobre todo sanitarios, educativos, culturales y turísticos reforzando la vitalidad de los núcleos de cabecera mediante la descentralización de los servicios en la medida de lo posible. Las inversiones en fomento del turismo resultan ridículas si pensamos el papel asignado a este tipo de actividad terciaria en toda clase de estudios sobre reactivación de la montaña, ya que el efecto sustitución que de otras rentas se asigna al turismo, queda anulado cuando se destina un tanto por ciento muy bajo del presupuesto anual. (Rubio y otros 1987).

En un sistema de economía de mercado donde la inversión selecciona los focos más desarrollados, es impensable que se produzca una inversión privada local en estas áreas deprimidas de montaña, si previamente no existe por parte de la Administracción una inversión voluntarista que opere como catalizador. Así posiblemente podria frenarse el largo proceso migratorio y el cambio de actividad económica en el Pirineo será beneficioso. 


\section{BIBLIOGRAFÍA}

Boserup, E., 1984: Población y Cambio tecnológico. Barcelona. Edif. Crítica.

Chadefaud, M. y Dalla Rosa, G., 1978: «La neige dans les Pyrénées Occidentales: enjeux et stratégies des collectivités locales". Revue géographique des Pyrénées et du Sud-Ouest. Tomo 49, Facs. 4, Toulouse, p. 477-515.

Dalla Rosa, G., 1980: “Vallee D'Aspe en Survie on les limites d'une politique en faveur de l'agriculture de montagne dans les Pyrénées françaises". Actes du colloogne franco-espagnol sur l'espace montagnard. Madrid, p. 633-652.

Daumas, M., 1976: La vie rurales dans le haut Aragón Oriental. Madrid. CSIC.

DEZERT, B., 1975: Activité industrielle et vie humaine en montagne. París, CDU.

Gabert, P. y Guichonnet, P., 1965: Les Alpes et les Stats alpins. París. PUF.

Gaviria, M., 1976: "Presente y futuro del Espacio Pirenáico". Actas del I Simposio. Zaragoza. Alcrudo editor.

LOPEZ DE SEBASTIÁN, J., 1981: “La gestión de la montaña: Colonización y Regeneración". Actas del Coloquio Hispano-Francés sobre las áreas de montaña. Madrid, MAPA.

MAJORAL, R., 1986: "La transición agrícola en áreas de montaña". Actas I Jornadas sobre alternativas de utilización del espacio en áreas de montaña. Huesca, CU, p. 163-188. 
MOLINA M., 1986: «El agua como recurso en las áreas de montaña». Actas I Jornadas sobre alternativas de utilización del espacio en áreas de montaña. Huesca, CU, p. 117-128.

MONTSERRAT, P. y FilLAT, F., 1979: "La ganadería extensiva y las culturas rurales montañesas». Anales del Instituto de Estudios Agropecuarios, n. ${ }^{\circ} 3$, Santander, p. 165-182.

NoIN, D., 1983: La transitión demographique dans le Monde. París. PUF.

PISANO, L. (a cura di), 1986: Cultura e Modernizzazione nei paesi di area Mediterránea. Milano. Franco Angeli.

Puigdefabregas, J., 1980: “La vida rural en la montaña española. Orientaciones para su promoción". Monografías del Instituto de Estudios Pirenáicos, n. $^{\circ}$ 107. Jaca.

PuYoL, R., 1979: Emigración y desigualdades regionales en España. Madrid. Emesa.

Rodríguez, J., 1985: Población y Territorio en España, siglos XIX y XX. Madrid, Espasa Calpe.

Rubio, M. T. y Castello, A., 1986: "Utilización del espacio en el Valle de Tena". Actas I Jornadas sobre alternativas de utilización del espacio en áreas de montaña. Huesca, CU, p. 227-255.

RuBio, M. T. y CASTELLO, A., 1987: «Turismo de invierno y ganadería como alternativas de aprovechamiento integral del espacio de montaña". Actas del / Congreso de Geografia Agraria. Canarias.

RuBıo, M. T. y otros, 1987: Costes de la reactivación económico-social en áreas deprimidas y de montaña en Aragón. Madrid. MOPU (inédito). 